Research Article

\title{
Oral Colonization of Staphylococcus Species in a Peritoneal Dialysis Population: A Possible Reservoir for PD-Related Infections?
}

\author{
Liliana Simões-Silva ${ }^{1},{ }^{1,2,3}$ Susana Ferreira, ${ }^{4}$ Carla Santos-Araujo, ${ }^{5,6}$ Margarida Tabaio, ${ }^{4}$ \\ Manuel Pestana $\mathbb{D}^{1,2,5,7}$ Isabel Soares-Silva $\mathbb{D}^{1,2}$ and Benedita Sampaio-Maia $\mathbb{D}^{1,2,4}$ \\ ${ }^{1}$ i3S-Instituto de Investigação e Inovação em Saúde, Universidade do Porto, Porto, Portugal \\ ${ }^{2}$ INEB-Instituto de Engenharia Biomédica, Universidade do Porto, Rua Alfredo Allen 208, 4200-180 Porto, Portugal \\ ${ }^{3}$ Faculdade de Medicina, Universidade do Porto, Porto, Portugal \\ ${ }^{4}$ Faculdade de Medicina Dentária, Universidade do Porto, Porto, Portugal \\ ${ }^{5}$ Departamento de Nefrologia, Centro Hospitalar de São João, EPE, Porto, Portugal \\ ${ }^{6}$ Departmento de Fisiologia e Cirurgia Cardiotorácica, Faculdade de Medicina, Centro de I $\backsim$ D Cardiovascular, \\ Universidade do Porto, Porto, Portugal \\ ${ }^{7}$ Departmento de Doenças Renais, Urológicas e Infecciosas, Faculdade de Medicina, Universidade do Porto, Porto, Portugal
}

Correspondence should be addressed to Benedita Sampaio-Maia; bmaia@fmd.up.pt

Received 19 April 2018; Accepted 11 June 2018; Published 2 August 2018

Academic Editor: Jorge Garbino

Copyright (c) 2018 Liliana Simões-Silva et al. This is an open access article distributed under the Creative Commons Attribution License, which permits unrestricted use, distribution, and reproduction in any medium, provided the original work is properly cited.

\begin{abstract}
Peritoneal dialysis-related infections are important morbidity/mortality causes, being staphylococci the most prevalent agents. Since Staphylococcus aureus nasopharynx carriage is a known risk factor for PD infections and the oral cavity is a starting point for systemic diseases development, we aimed at comparing the oral staphylococci colonization between PD patients and controls and studying the association with PD-related infections. Saliva samples were plated in Mannitol salt, and isolates were identified by DnaJ gene sequencing. Staphylococci PD-related infections were recorded throughout the 4-year period following sample collection. Staphylococcus colonization was present in $>90 \%$ of the samples from both groups (a total of nine species identified). PD patients presented less diversity and less prevalence of multispecies Staphylococcus colonization. Although all patients presenting Staphylococcus epidermidis PD-related infections were also colonized in the oral cavity by the same agent, only 1 out of 7 patients with ESI caused by S. aureus presented S. aureus oral colonization. Staphylococci are highly prevalent in the oral cavity of both groups, although PD patients presented less species diversity. The association between oral Staphylococcus carriage and PD-related infections was present for S. epidermidis but was almost inexistent for S. aureus, so, further studies are still necessary to evaluate the infectious potential of oral Staphylococcus carriage in PD.
\end{abstract}

\section{Introduction}

Peritoneal dialysis (PD) is a home-based renal replacement therapy for end-stage chronic kidney disease (CKD) patients, presenting significantly lower costs and higher satisfaction levels in comparison to hemodialysis (HD) [1]. Nonetheless, infection remains an important cause of morbidity and mortality among PD patients $[1,2]$. Peritonitis and exit-site infections (ESI) are the more relevant and common PD-related infections, and Staphylococcus species are the most frequent etiological agents responsible for both types of infection [3, 4]. Worldwide, Staphylococcus epidermidis and other coagulase-negative Staphylococcus (CNS) are the most prevalent PD-related infectious agents. On the contrary, Staphylococcus aureus is associated with more severe PDrelated infections leading to increased risks of hospitalization, death, and catheter removal $[2,5,6]$. Staphylococcus haemolyticus, Staphylococcus warneri, Staphylococcus hominis, 
Staphylococcus simulans, Staphylococcus capitis, and Staphylococcus saprophyticus are staphylococci species also reported as PD-related infectious agents $[7,8]$.

It is assumed that contamination at the time of PD fluid exchange is a major cause of peritonitis, occurring mainly by an external route [9]. For this reason, general measures concerning exit-site care, such as meticulous hand hygiene and face-mask wear are recommended during the dialysis exchange by the International Society for Peritoneal Dialysis (ISPD) [10]. Other measure is the prophylactic use of topical antibiotic treatment of PD catheter exit-site, known to reduce ESI $[4,11]$, although its effect on peritonitis rate is not clear $[4,11,12]$. Also, the screening for nasal $S$. aureus carriage prior to PD catheter insertion is recommended and, if positive, treatment with topical nasal application of mupirocin is recommended [13]. The efficacy of this prophylactic intranasal antibiotic treatment has been showed in several prospective studies [14-16].

Another less explored route of infection in PD patients is the haematogenous pathway. It is known that oral microorganisms are frequently responsible for bacteraemia due to routine daily hygiene activities, such as tooth brushing and dental tape use or invasive dental procedures [17, 18]. For that reason, risk patients including those on $\mathrm{PD}$ therapy should receive prophylactic antibiotic treatment before dental interventions [17]. Although it is recognized that the oral cavity can be a starting point for dissemination of pathogenic organisms, by both external and haematogenous routes, to our knowledge, there are no studies linking the oral microbiome and PD-related infections. Thus, the aim of the present study was to assess the oral colonization with Staphylococcus spp. in PD patients in comparison with healthy controls, and explore in patients on PD therapy the association between oral colonization with Staphylococcus spp. and PD-related infections.

\section{Materials and Methods}

Twenty-one end-stage CKD adult patients undergoing PD therapy followed up at the outpatient clinic of the Nephrology Department of the "Centro Hospitalar de S. João" for more than one month were invited to participate in the study. A convenience sample was obtained related with the attendance of patients to the outpatient clinic during a period of 6 months. The control group included 14 adult healthy subjects recruited among family members of PD patients, to gather individuals with the same socioeconomic and environmental conditions as PD patients. The exclusion criteria were recent history of infection (less than 1 month), inability to give informed consent, pregnancy, and severe acute illness. The study protocol was approved by the Ethics Committee for Health and Institutional Review Board of São João Hospital Centre.

Demographic and relevant clinical information was gathered for each patient including age, gender, smoking habits, education level, blood pressure, aetiology of renal disease, residual renal function, time on $\mathrm{PD}$, and infectious complications during PD. Demographic information was also gathered for the control healthy population, namely, age, gender, smoking habits, and education level.
2.1. Oral Evaluation. Oral hygiene was assessed in both groups using the visual plaque indexes (VPI) in four sites of each tooth (mesiobuccal, midbuccal, distobuccal, and midlingual); the percentage of the examined sites with visible plaque ranged from $0 \%$ to $100 \%$.

Additionally, before the oral clinical evaluation, unstimulated whole saliva was collected from both groups into a sterile container for 5 minutes in a quiet room, between 9:00 to 11:00 AM to minimize the circadian rhythm effects and at least $2 \mathrm{~h}$ after eating, tooth brushing, mouth washing, or smoking. Immediately after collection, the saliva was stored at $-20^{\circ} \mathrm{C}$ for biochemical analysis or mixed $1: 1$ in Brain Heart Infusion with 20\% glycerol for microbial analysis and cryopreserved at $-80^{\circ} \mathrm{C}$. The total volume collected over a $5 \mathrm{~min}$ period was registered, for saliva flow rate $(\mathrm{mL} / \mathrm{min})$ determination. The saliva $\mathrm{pH}$ was also measured immediately after collection using $\mathrm{pH}$ strips (5.0-8.0, Duotest, Germany). The saliva urea concentration was determined by an enzymatic UV test (method "ureaseGLDH”) and quantified by an automatic analyser, Pentra C200 (Horiba ABX Diagnostics, Switzerland).

2.2. Staphylococcus spp. Isolation. Staphylococcus was isolated from saliva from both groups and quantified. Saliva samples were serially diluted with $0.9 \%$ sterile $\mathrm{NaCl}$ solution and plated in triplicate in a selective and differential culture medium, Mannitol salt agar (Liofilchem, Italy). Plates were incubated aerobically for $48 \mathrm{~h}$ at $37^{\circ} \mathrm{C}$. The total number of colonies was determined, and the quantification results were expressed in a logarithmic scale of colony-forming units per $\mathrm{ml}$ of saliva $\left(\log _{10} \mathrm{CFU} / \mathrm{ml}\right)$. The lower limit of detection was $10^{2} \mathrm{CFU} / \mathrm{ml}$. The Gram-positive and catalase-positive isolates were purified by reisolation in BHI (Biolab Inc, Budapest, Hungary) agar and stored in BHI containing 10\% glycerol at $-80^{\circ} \mathrm{C}$. The isolated colonies were identified using the DnaJ gene amplification and sequencing approach.

\subsection{Staphylococcus Identification by DnaJ Gene Amplification.} DNA was extracted using the boiling method; briefly, 5 colonies were suspended in $100 \mu \mathrm{L}$ of molecular grade water and incubated at $100^{\circ} \mathrm{C}$ for 10 minutes. The diluted suspension ( 1 : 10) was centrifuged at $12,000 \mathrm{rpm}$ for $10 \mathrm{~min}$, and the supernatant was used as a DNA template. DnaJ gene was amplified with the DFS Master Mix (Bioron, Germany) according to the manufacturer's instructions using the primers Staph_forward, 5'-GCCAAAAGAGACTATTATGA-3' ${ }^{\prime}$, and Staph_reverse, $5^{\prime}$-ATTGYTTACCYGTTTGTGTACC-3' and the amplification conditions previously described, with MyCycler Thermal Cycler (Bio-Rad, California, USA) [19]. PCR products were visualized by electrophoresis on a $1 \%$ agarose gel (SeaKem ${ }^{\circledR}$ LE Agarose; Lonza, Cologne, Germany) with GelRed $^{\mathrm{TM}}$ (GeneON, Ludwigshafen am Rhein, Germany) and then visualized in ChemiDoc XR (Bio-Rad, California, USA). PCR amplification products were identified by sequencing (StabVida, Caparica, Portugal). The identification by sequencing of DnaJ gene was only considered when the sequencing identity was $98 \%$ or higher to DnaJ sequences or 
sequences resulting from whole genome sequencing deposited at GenBank [20].

2.4. PD-Related Infection Episodes. The PD-related infections by Staphylococcus spp. occurring from the start of PD therapy up to 4 years after sample collection were registered in all 21 studied PD patients. However, due to PD technique dropout, 20 patients remained in the first follow-up year, 19 in the second, 14 in the third, and 11 in the fourth.

2.5. Data Analysis. Data were analysed using IBM SPSS Statistics for Windows, Version 23.0 (IBM Corp, NY, USA). The categorical variables were described through relative frequencies (\%) and analysed by the chi-square independence test or Fisher Exact test when more than 1 cell had expected counts less than 5 . Continuous variables were described using mean \pm standard deviation (SD) and analysed by Student's $t$-test. A $P$ value of less than 0.05 was assumed to denote a statistically significant difference.

\section{Results}

Clinical information of PD patients is included in Table 1, namely, aetiology of $\mathrm{CKD}, \mathrm{PD}$ vintage, residual renal function, and blood pressure. The most prevalent aetiologies of CKD were glomerular disease, including diabetic nephropathy, and tubulointerstitial disease, namely, polycystic kidney disease. At sample collection, the average time on PD therapy was $15.45 \pm 16.90$ months, ranging from 1 to 72 months.

Demographic information, namely, age, gender, educational level, smoking habits, parameters regarding oral hygiene, and saliva biochemistry of both groups is included in Table 2. The two groups presented similar demographic characteristics. Also, there were no significant differences between the two groups in the education level and smoking habits. Both groups had high VPI values, revealing a low oral hygiene status. Regarding saliva biochemical parameters, the only significant differences were in the saliva $\mathrm{pH}$ and urea concentration, being both parameters higher in PD patients when compared to the control group.

The PD patients and healthy controls presented similar total load (CFU/mL) of Staphylococcus in saliva (Table 3). Nine Staphylococcus species were identified in the saliva from both groups, namely, S. epidermidis, S. aureus, S. capitis, S. saprophyticus, S. hominis, Staphylococcus cohnii, Staphylococcus pasteuri, Staphylococcus lugdunensis, and S. warneri (Supplementary Table 1).

No significant differences were observed in the prevalence of the different Staphylococcus species between the two groups. However, the prevalence of more than one Staphylococcus species (multispecies) in saliva was significantly higher in the control group than in PD patients (Table 4). When analyzing the colonization status of the family members of PD patients (10 pairs), we found that 6 patientfamily member pairs presented oral colonization by $S$. epidermidis. One of these pairs also presented colonization by $S$. saprophyticus.
TABle 1: Aetiology of chronic kidney disease (CKD), peritoneal dialysis (PD) vintage, residual renal function, and blood pressure of PD patients.

\begin{tabular}{lc}
\hline & PD patients \\
\hline Aetiology of CKD & \\
Glomerular disease & $52.38 \%$ \\
Diabetic nephropathy & $19.05 \%$ \\
Other glomerular diseases & $33.33 \%$ \\
Tubulointerstitial disease & $23.81 \%$ \\
$\quad$ Autosomal dominant polycystic & $14.29 \%$ \\
kidney disease & $9.52 \%$ \\
$\quad$ Other tubulointerstitial diseases & $23.81 \%$ \\
Unknown & $15.45 \pm 16.90$ \\
PD vintage (months) & $6.98 \pm 4.52$ \\
Residual renal function (mL/min) & \\
Blood pressure & $130.19 \pm 19.65$ \\
Systolic & $78.29 \pm 10.96$ \\
Diastolic
\end{tabular}

Results are shown in prevalence (\%); CKD, chronic kidney disease; PD, peritoneal dialysis.

TABLE 2: Demographic information and parameters regarding oral hygiene of peritoneal dialysis (PD) patients and healthy controls.

\begin{tabular}{lccc}
\hline & PD patients & Controls & $P$ value \\
\hline Age (years) & $46.8 \pm 9.7$ & $42.2 \pm 14.5$ & 0.282 \\
Sex (male \%) & $42.9 \%$ & $28.6 \%$ & 0.392 \\
Education level & & & $>0.999$ \\
$\quad$ Basic/elementary school & $85.7 \%$ & $83.3 \%$ & \\
$\quad$ High school/university & $14.3 \%$ & $16.7 \%$ & \\
Smoking habits & & & \\
$\quad$ Past & $58.3 \%$ & $33.3 \%$ & 0.387 \\
$\quad$ At sample collection & $16.7 \%$ & $11.1 \%$ & $>0.999$ \\
Visual plaque index (\%) & $59.7 \pm 27.7$ & $48.4 \pm 23.1$ & 0.254 \\
Saliva biochemistry & & & \\
$\quad$ Flow rate (mL/min) & $0.40 \pm 0.26$ & $0.41 \pm 0.30$ & 0.871 \\
$\quad$ pH & $7.72 \pm 0.53$ & $7.06 \pm 0.39$ & $<\mathbf{0 . 0 0 1}$ \\
$\quad$ Urea (mg/dL) & $110.41 \pm 36.64$ & $28.79 \pm 5.90$ & $<\mathbf{0 . 0 0 1}$ \\
\hline
\end{tabular}

Results are shown in prevalence (\%) or mean \pm SD; PD, peritoneal dialysis.

TABle 3: Prevalence and quantification of Staphylococcus spp. in the oral cavity of PD patients and controls.

\begin{tabular}{lccc}
\hline & PD patients & Controls & $P$ value \\
\hline Prevalence & $90.5 \%$ & $92.9 \%$ & $>0.999$ \\
Counts $\left(\log _{10} \mathrm{CFU} / \mathrm{mL}\right)$ & $2.79 \pm 0.60$ & $2.43 \pm 0.64$ & 0.120 \\
\hline
\end{tabular}

Results are prevalence (\%) or mean $\pm \mathrm{SD}$; PD, peritoneal dialysis; $\mathrm{CFU}$, colony-forming units.

The occurrence of PD-related Staphylococcus infectious episodes was retrieved from clinical records from the date of entrance in the PD program until PD dropout or the end of the 4 th year after oral sample collection, accounting for an average of $53.9 \pm 27.8$ months. The total number of patients that had at least one infection episode, peritonitis and/or ESI, is presented in Table 5, whereas in Table 6, the total number of infection episodes and the Staphylococcus species detected are presented.

The association between the PD-related infections by staphylococci and the oral colonization with the same 
TABle 4: Prevalence of Staphylococcus species identified in isolates from saliva of peritoneal dialysis (PD) patients and controls.

\begin{tabular}{lccc}
\hline & PD patients & Controls & $P$ value \\
\hline Staphylococcus species & & & \\
S. epidermidis & $89.5 \%$ & $92.3 \%$ & $>0.999$ \\
S. aureus spp. aureus & $21.1 \%$ & $23.1 \%$ & $>0.999$ \\
S. capitis & $15.8 \%$ & $7.7 \%$ & 0.629 \\
S. saprophyticus & $10.5 \%$ & $15.4 \%$ & $>0.999$ \\
S. hominis & $5.3 \%$ & $23.1 \%$ & 0.279 \\
S. cohnii spp. cohnii & $5.3 \%$ & $0 \%$ & $>0.999$ \\
S. pasteuri & $5.3 \%$ & $7.7 \%$ & $>0.999$ \\
S. lugdunensis & $0 \%$ & $7.7 \%$ & 0.406 \\
S. warneri & $0 \%$ & $15.4 \%$ & 0.157 \\
Multispecies & $36.8 \%$ & $76.9 \%$ & $\mathbf{0 . 0 2 6}$ \\
\hline
\end{tabular}

Results are prevalence (\%); PD, peritoneal dialysis; ${ }^{*} P<0.05$.

TABLE 5: Total number of PD patients with one or more infectious episodes, peritonitis, and/or exit-site infections (ESI), in particular caused by Staphylococcus spp.

\begin{tabular}{lcccc}
\hline & \multicolumn{2}{c}{$\begin{array}{c}\text { Peritonitis } \\
\text { Ttaphylococcus } \\
\text { spp. }\end{array}$} & Total & $\begin{array}{c}\text { ESI } \\
\text { Staphylococcus } \\
\text { spp. }\end{array}$ \\
\hline $\begin{array}{l}\text { PD patients } \\
\text { with }\end{array}$ & 10 & 5 & 19 & 12 \\
$\begin{array}{l}\text { infectious } \\
\text { episodes } \\
(n=19)\end{array}$ & $(47.6 \%)$ & $(23.8 \%)$ & $(90.5 \%)$ & $(57.1 \%)$ \\
\hline
\end{tabular}

TABle 6: Total number of infectious episodes and Staphylococcus species identified in peritonitis and exit-site infections (ESI) of PD patients.

\begin{tabular}{lcc}
\hline \multirow{2}{*}{ Infectious agent } & \multicolumn{2}{c}{ PD-related infections $(n=114)$} \\
& Peritonitis $(n=23)$ & ESI $(n=91)$ \\
\hline Others & $16(69.6 \%)$ & $63(69.2 \%)$ \\
Staphylococci & $7(\mathbf{3 0 . 4 \% )}$ & $\mathbf{2 8 ~ ( 3 0 . 8 \% )}$ \\
S. epidermidis & 5 & 9 \\
S. aureus & 1 & 16 \\
S. warneri & 1 & 1 \\
S. hominis & 0 & 1 \\
Nonidentified CNS & 0 & 1 \\
\hline
\end{tabular}

CNS, coagulase-negative Staphylococcus.

Staphylococcus species was examined (Figure 1). All PD patients with peritonitis or ESI caused by S. epidermidis were colonized by the same species in the oral cavity (Table 7). Among the PD patients with ESI caused by $S$. aureus, only 1 was colonized with the same species in the oral cavity (Table 7). Moreover, the only patient with S. aureus peritonitis was also not orally colonized with the same species. Oral colonization of PD patients with multispecies staphylococci was not associated to a higher prevalence of PDrelated infections $(P>0.05)$.

\section{Discussion}

In the Human Oral Microbiome Database (http://www.homd. org/), few Staphylococcus species are described, namely,
S. aureus, S. epidermidis, S. warneri, and Staphylococcus caprae. Our results provide evidence for the presence of six other Staphylococcus species, namely, S. saprophyticus, S. capitis, S. cohnii, S. pasteuri, S. hominis, and S. lugdunensis. Taking in consideration that we found a prevalence close to $100 \%$ of Staphylococcus in both PD patients and control groups, we suggest that this genus is a common member of the oral microbiome. In agreement, other authors also described Staphylococcus species as frequent oral colonizers of adults, in health and oral disease $[21,22]$. Nonetheless, our study was the first to report $S$. pasteuri, considered a rare CNS clinical isolate [23], as a member of the oral microbiome.

Although staphylococci are major pathogens of PDrelated infections, there are no studies exploring the oral microbiome as a source of infection in PD patients. The likelihood that these microorganisms could act as opportunistic infectious agents to distant body locations triggered our need to better understand the oral colonization status of PD patients. Overall, we did not observe differences between the two groups in specific species oral colonization, although the oral microbiota of PD patients presented lower staphylococci diversity. This lower diversity in $\mathrm{PD}$ patients may be justified by more frequent antibiotic therapy of these patients, as well as by changes in the oral biochemical milieu, including higher $\mathrm{pH}$ and urea content observed in patients on PD therapy.

Although infections are a major concern to PD patients, risk factors and transmission routes are far from being fully understood. Given that many preventive measures are in place to prevent PD-related infections by external route, such as mask use and prophylactic use of topical antibiotic treatment of PD catheter exit-site $[4,10,11]$, staphylococci transmission by a haematogenous route should be considered. In line with this, recent studies demonstrate that the uterine microbiome is more similar to the oral microbiome than to the vaginal, intestinal, or skin microbiome [24, 25], which reinforces the role of the haematogenous route in the transmission of oral microorganisms.

In agreement with previous reports, the Staphylococcus species identified as agents of PD-related infections in our group of PD patients were $S$. aureus, S. epidermidis, $S$. warneri, and $S$. hominis $[7,8,26]$. In the present study, all the patients with $S$. epidermidis infections, either peritonitis or ESI, were colonized orally by the same species suggesting that the oral cavity may have the potential to behave as a reservoir of PD-related infections induced by these agents. However, S. epidermidis is a highly ubiquitous microorganism in human skin and mucosa, and so, the phylogenetic relatedness between common species would be an important asset to determine the real transmission route. Moreover, only 1 out of 7 patients with $S$. aureus ESI was colonized orally by $S$. aureus thus suggesting that the oral cavity may not behave as a potential reservoir of PD-related infections induced by $S$. aureus specifically. These partial contradictory results, together with the small sample size restricted to a single-centre, hinder us to draw a conclusion about the potential of the oral cavity as a reservoir of PD-related pathogens. Further studies are thus necessary to clarify this hypothesis. 


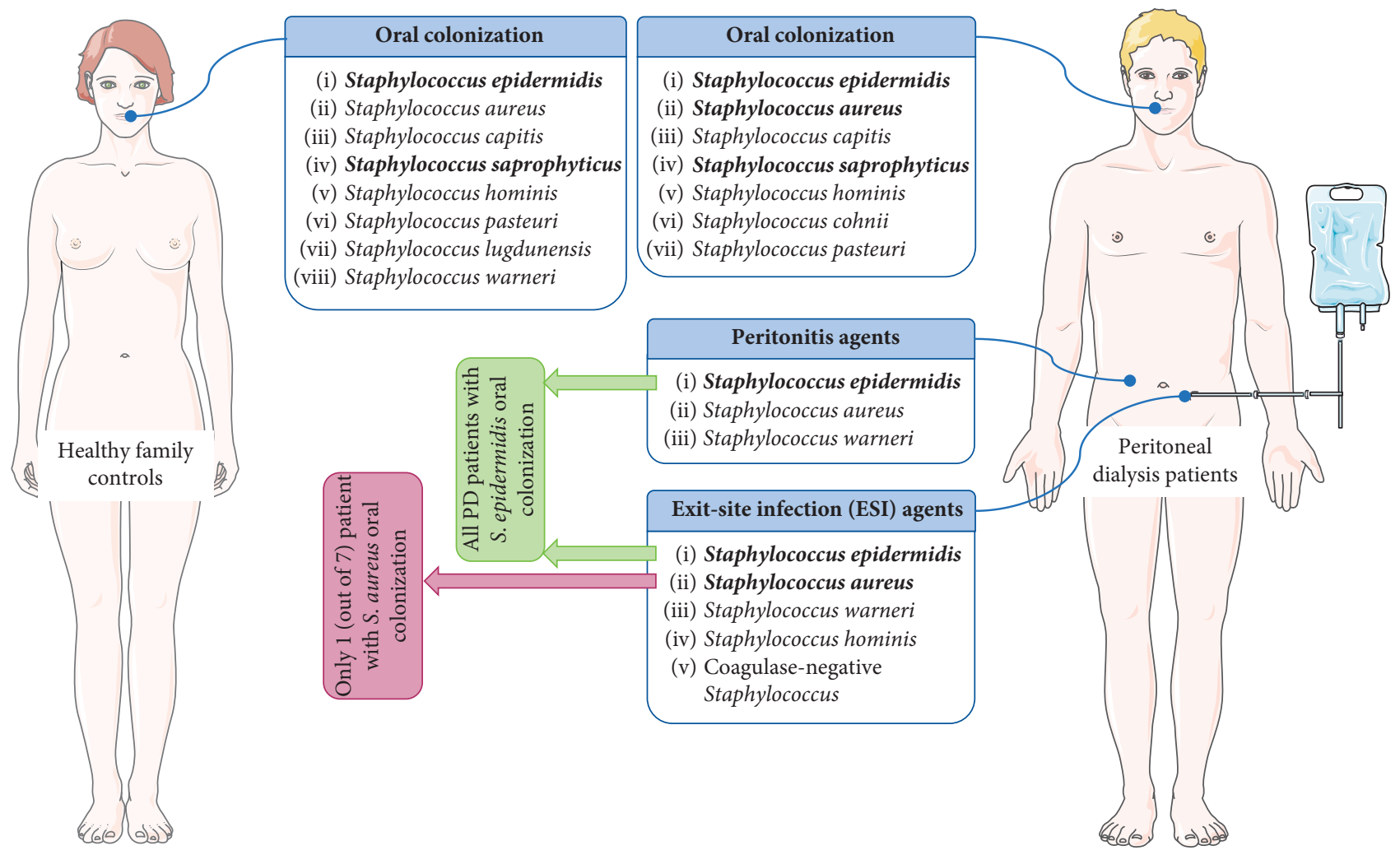

FIGURE 1: Staphylococcus oral colonization of PD patients and healthy controls and Staphylococcus agents responsible for PD-related infections. Common species are in bold. Figure was produced using Servier Medical Art, http://www.servier.com/Powerpoint-image-bank.

TABLE 7: Comparison between peritoneal infections, peritonitis, or exit-site infections (ESI) caused by Staphylococcus species and oral colonization of PD patients.

\begin{tabular}{lcccc}
\hline & \multicolumn{2}{c}{ Infectious agent } & \multicolumn{2}{c}{$\begin{array}{c}\text { Oral } \\
\text { colonization }\end{array}$} \\
& ESI & Peritonitis & ESI & Peritonitis \\
\hline Staphylococcus epidermidis & 6 & 3 & 6 & 3 \\
Staphylococcus aureus & 7 & 1 & 1 & 0 \\
\hline
\end{tabular}

\section{Conclusion}

Staphylococci are highly prevalent in the oral cavity of both groups, although PD patients presented less diversity of species in comparison to controls. Given that the association between oral Staphylococcus carriage and PD-related infections was present for S. epidermidis but was almost inexistent for $S$. aureus, further studies are necessary to evaluate the relationship between oral Staphylococcus carriage and $\mathrm{PD}$-related infections development.

\section{Data Availability}

The data used to support the findings of this study are available from the corresponding author upon request.

\section{Ethical Approval}

All procedures performed in studies involving human participants were in accordance with the ethical standards of the institutional and/or national research committee at which the studies were conducted (Ethics Committee for Health and Institutional Review Board of São João Hospital Centre approval number 159/11) and with the 1964 Helsinki Declaration and its later amendments or comparable ethical standards.

\section{Consent}

Informed consent was obtained from all individual participants included in the study.

\section{Conflicts of Interest}

The authors declare that there are no conflicts of interest regarding the publication of this article.

\section{Acknowledgments}

The authors thank the nurse Maria João Sousa, Department of Nephrology, São João Hospital Center, EPE, for helping in sample collection and Joana Sousa from the Faculty of Dental Medicine for helping with the oral evaluation. Liliana SimõesSilva was supported by SFRH/BD/84837/2012 and Isabel Soares-Silva was supported by SFRH/BPD/101016/2014 from FCT/QREN-POPH/FSE. This work was financed by Fundo Europeu de Desenvolvimento Regional (FEDER) funds through the COMPETE 2020-Operacional Programme for Competitiveness and Internationalisation (POCI), Portugal 2020; by Portuguese funds through Fundação para a Ciência e 
a Tecnologia (FCT)/Ministério da Ciência, Tecnologia e Inovação, in the framework of the project "Institute for Research and Innovation in Health Sciences" (POCI-01-0145FEDER-007274 and POCI-01-0145-FEDER-029777); by the project NORTE-01-0145-FEDER-000012, supported by Norte Portugal Regional Operational Programme (NORTE 2020), under the PORTUGAL 2020 Partnership Agreement, through the European Regional Development Fund (ERDF); and by IJUP Projects, University of Porto.

\section{Supplementary Materials}

Supplementary Table 1 presents the Staphylococcus species identified in PD patients and controls, as well as the number of individuals where each of the Staphylococcus species was identified. (Supplementary Materials)

\section{References}

[1] R. Sinnakirouchenan and J. L. Holley, "Peritoneal dialysis versus hemodialysis: risks, benefits, and access issues," Advances in Chronic Kidney Disease, vol. 18, no. 6, pp. 428-432, 2011.

[2] P. K. Li and K. M. Chow, "Infectious complications in dialysis-epidemiology and outcomes," Nature Reviews $\mathrm{Ne}$ phrology, vol. 8, no. 2, pp. 77-88, 2011.

[3] A. Rocha, A. Rodrigues, L. Teixeira, M. J. Carvalho, D. Mendonca, and A. Cabrita, "Temporal trends in peritonitis rates, microbiology and outcomes: the major clinical complication of peritoneal dialysis," Blood Purification, vol. 33, no. 4, pp. 284-291, 2012.

[4] G. Xu, W. Tu, and C. Xu, "Mupirocin for preventing exit-site infection and peritonitis in patients undergoing peritoneal dialysis," Nephrology Dialysis Transplantation, vol. 25, no. 2, pp. 587-592, 2010.

[5] S. A. Zelenitsky, J. Howarth, P. Lagace-Wiens et al., "Microbiological trends and antimicrobial resistance in peritoneal dialysis-related peritonitis, 2005 to 2014," Peritoneal Dialysis International, vol. 3, no. 2, pp. 170-176, 2016.

[6] S. J. Nessim, P. Komenda, C. Rigatto, M. Verrelli, and M. M. Sood, "Frequency and microbiology of peritonitis and exit-site infection among obese peritoneal dialysis patients," Peritoneal Dialysis International, vol. 33, no. 2, pp. 167-174, 2013.

[7] M. H. Wilcox, P. Williams, D. G. Smith, B. Modun, R. G. Finch, and S. P. Denyer, "Variation in the expression of cell envelope proteins of coagulase-negative staphylococci cultured under iron-restricted conditions in human peritoneal dialysate," Journal of General Microbiology, vol. 137, no. 11, pp. 2561-2570, 1991.

[8] J. H. Shin, S. H. Kim, H. S. Jeong et al., "Identification of coagulase-negative staphylococci isolated from continuous ambulatory peritoneal dialysis fluid using $16 \mathrm{~S}$ ribosomal RNA, tuf, and SodA gene sequencing," Peritoneal Dialysis International, vol. 31, no. 3, pp. 340-346, 2011.

[9] T. A. Golper, M. E. Brier, M. Bunke et al., "Risk factors for peritonitis in long-term peritoneal dialysis: the network 9 peritonitis and catheter survival studies," American Journal of Kidney Diseases, vol. 28, no. 3, pp. 428-436, 1996.

[10] P. K. Li, C. C. Szeto, B. Piraino et al., "ISPD peritonitis recommendations: 2016 update on prevention and treatment," Peritoneal Dialysis International, vol. 36, no. 5, pp. 481-508, 2016.
[11] C. Grothe, M. Taminato, A. Belasco, R. Sesso, and D. Barbosa, "Prophylactic treatment of chronic renal disease in patients undergoing peritoneal dialysis and colonized by Staphylococcus aureus: a systematic review and meta-analysis," BMC Nephrology, vol. 17, no. 1, p. 115, 2016.

[12] J. Ritzau, R. M. Hoffman, and A. H. Tzamaloukas, "Effect of preventing Staphylococcus aureus carriage on rates of peritoneal catheter-related staphylococcal infections. Literature synthesis," Peritoneal Dialysis International, vol. 21, no. 5, pp. 471-479, 2001.

[13] C. C. Szeto, P. K. Li, D. W. Johnson et al., "ISPD catheterrelated infection recommendations: 2017 update," Peritoneal Dialysis International, vol. 37, no. 2, pp. 141-154, 2017.

[14] M. Perez-Fontan, M. Rosales, A. Rodriguez-Carmona et al., "Treatment of Staphylococcus aureus nasal carriers in CAPD with mupirocin," Advances in Peritoneal Dialysis, vol. 8, pp. 242-245, 1992.

[15] The Mupirocin Study Group, "Nasal mupirocin prevents Staphylococcus aureus exit-site infection during peritoneal dialysis," Journal of the American Society of Nephrology, vol. 7, no. 11, pp. 2403-2408, 1996.

[16] R. Sesso, K. Parisio, A. Dalboni et al., "Effect of sodium fusidate and ofloxacin on Staphylococcus aureus colonization and infection in patients on continuous ambulatory peritoneal dialysis," Clinical Nephrology, vol. 41, no. 6, pp. 370-376, 1994.

[17] F. K. Bahrani-Mougeot, B. J. Paster, S. Coleman, J. Ashar, S. Barbuto, and P. B. Lockhart, "Diverse and novel oral bacterial species in blood following dental procedures," Journal of Clinical Microbiology, vol. 46, no. 6, pp. 2129-2132, 2008.

[18] B. Sampaio-Maia, I. M. Caldas, M. L. Pereira, D. PérezMongiovi, and R. Araujo, "The oral microbiome in health and its implication in oral and systemic diseases," Advances in Applied Microbiology, vol. 97, pp. 171-210, 2016.

[19] M. M. Shah, H. Iihara, M. Noda et al., "dnaJ gene sequencebased assay for species identification and phylogenetic grouping in the genus Staphylococcus," International Journal of Systematic and Evolutionary Microbiology, vol. 57, no. 1, pp. 25-30, 2007.

[20] D. A. Benson, M. Cavanaugh, K. Clark et al., "GenBank," Nucleic Acids Research, vol. 45, no. D1, pp. D36-D42, 2013.

[21] R. Percival, S. Challacombe, and P. Marsh, "Age-related micro-biological changes in the salivary and plaque micro flora of healthy adults," Journal of Medical Microbiology, vol. 35, no. 1, pp. 5-11, 1991.

[22] A. J. Smith, M. S. Jackson, and J. Bagg, "The ecology of Staphylococcus species in the oral cavity," Journal of Medical Microbiology, vol. 50, no. 11, pp. 940-946, 2001.

[23] R. Morfin-Otero, M. A. Martinez-Vazquez, D. Lopez, E. Rodriguez-Noriega, and E. Garza-Gonzalez, "Isolation of rare coagulase-negative isolates in immunocompromised patients: Staphylococcus gallinarum, Staphylococcus pettenkoferi and Staphylococcus pasteuri," Annals of Clinical and Laboratory Science, vol. 42, no. 2, pp. 182-185, 2012.

[24] K. Aagaard, J. Ma, K. M. Antony, R. Ganu, J. Petrosino, and J. Versalovic, "The placenta harbors a unique microbiome," Science Translational Medicine, vol. 6, no. 237, p. 237ra65, 2014.

[25] M. S. Payne and S. Bayatibojakhi, "Exploring preterm birth as a polymicrobial disease: an overview of the uterine microbiome," Frontiers in Immunology, vol. 5, p. 595, 2014.

[26] B. Piraino, G. R. Bailie, J. Bernardini et al., "Peritoneal dialysis-related infections recommendations: 2005 update," Peritoneal Dialysis International, vol. 25, no. 2, pp. 107-131, 2005. 


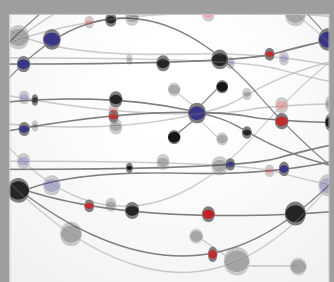

The Scientific World Journal
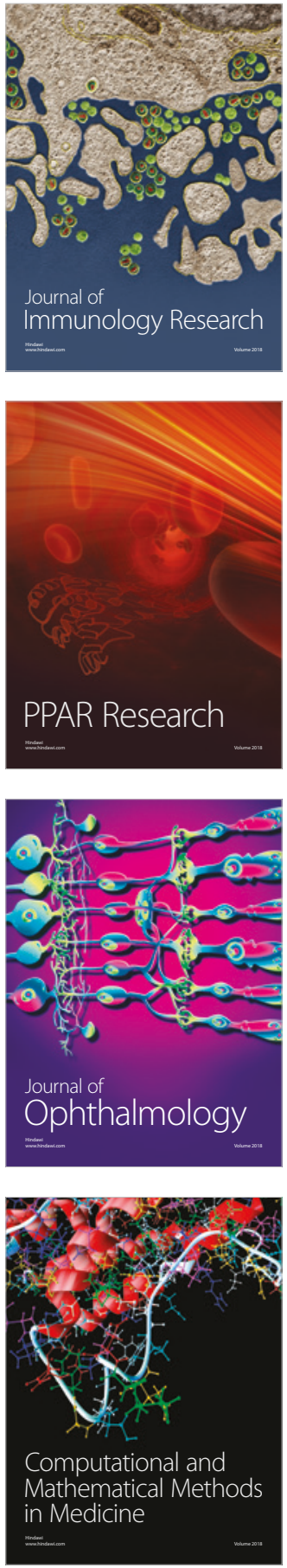

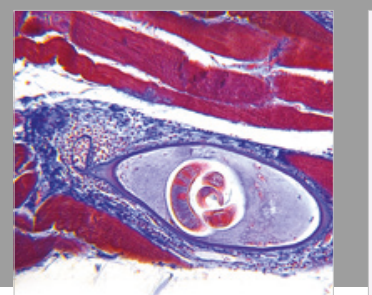

Gastroenterology Research and Practice

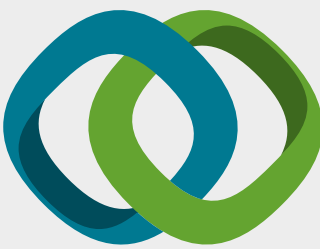

\section{Hindawi}

Submit your manuscripts at

www.hindawi.com
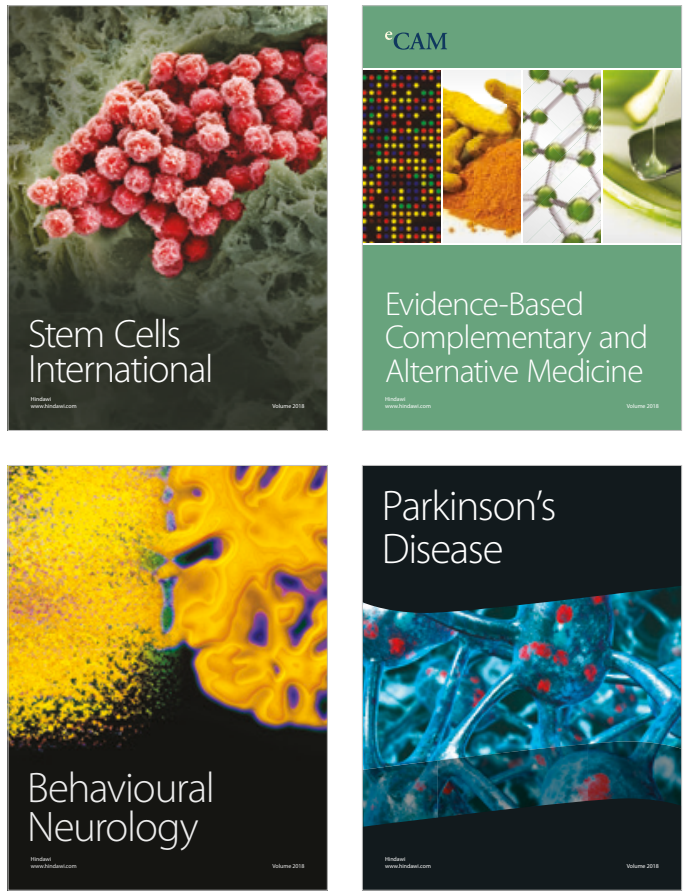

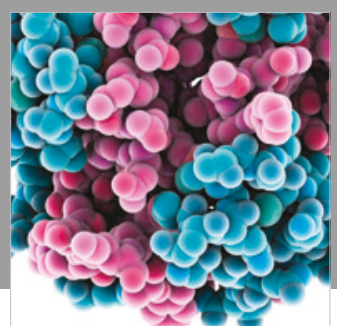

ournal of

Diabetes Research

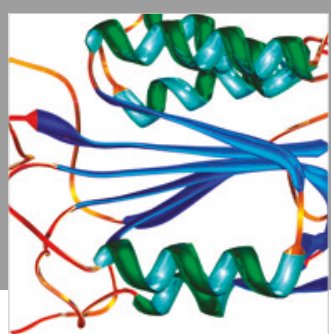

Disease Markers
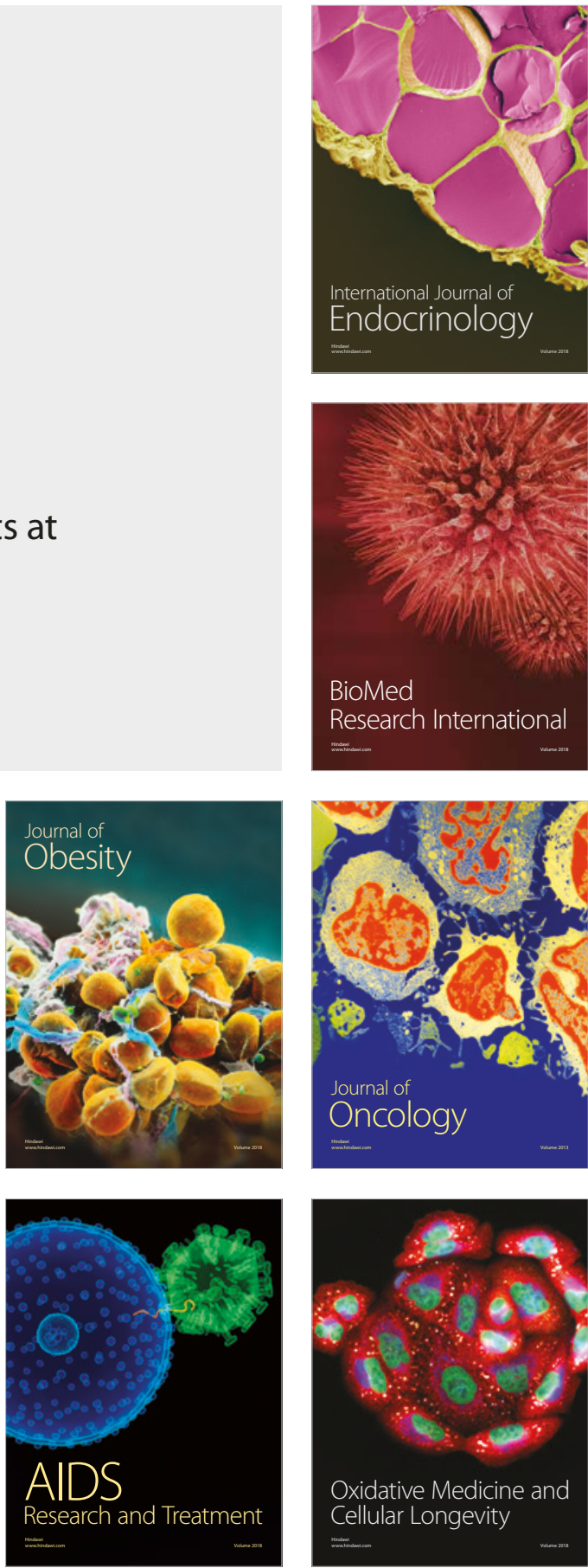